Doug Geisler, Eva K. Grebel, and Dante Minniti, eds.

\title{
Building Dwarf Galaxies out of Merged Young Star Clusters
}

\author{
Michael Fellhauer \\ Astronomisches Rechen-Institut, Mönchhofstr. 12-14, 69120 Heidelberg, \\ Germany
}

\begin{abstract}
Young star clusters in interacting galaxies are often found in groups or clusters of star clusters containing up to 100 single clusters. In our project we study the future fate of these clusters of star clusters. We find that the star clusters merge on time scales of a few dynamical crossing times of the super-cluster. The resulting merger object has similarities with observed dwarf ellipticals (dE). Furthermore, if destructive processes like tidal heating, dynamical friction or interaction with disc or bulge of the parent galaxy are taken into account our merger objects may evolve into objects resembling dwarf spheroidal galaxies (dSph), without the need of a high dark matter content.
\end{abstract}

\section{Introduction}

Systems of interacting galaxies (like The Antennae or Stephan's Quintet) show intense star formation. In the tidal tails one finds very young, massive star clusters $\left(R_{\mathrm{eff}} \approx 2-10 \mathrm{pc} ; M \approx 10^{4}-10^{7} \mathrm{M}_{\odot}\right.$; see e.g. Whitmore et al. (1999)). These star clusters are not distributed uniformly but are clustered into groups, some containing more than a hundred clusters. Here we investigate the future fate of these clusters of star clusters ("super-clusters"). Following the arguments of Kroupa (1998) these super-clusters may be bound entities, i.e. the star clusters in the super-cluster are not only bound to the tidal field of the parent galaxy but also bound to each other. Therefore, the star clusters are very likely to merge with each other on a few dynamical times of the super-cluster. This merging process and the resulting merger object are the topic of this project.

\section{Setup}

We simulate super-clusters containing $N_{0}=20$ star clusters. Each of them is represented by a Plummer sphere, containing 100,000 particles, a total mass of $10^{6} \mathrm{M}_{\odot}$, a scale-length (Plummer radius) of $R_{\mathrm{pl}}=6 \mathrm{pc}$ and a crossing time of 1.4 Myr. All star clusters are distributed into an encompassing Plummer sphere with varied scale-length, $R_{\mathrm{pl}}^{\mathrm{sc}}=50,75,150,300 \mathrm{pc}$, and this super-cluster is placed on a circular orbit at distance $D=5,10,20,30,50,100 \mathrm{kpc}$ around an analytic galactic potential with an asymptotically flat rotation curve with $v_{\mathrm{c}}=220 \mathrm{~km} / \mathrm{s}$ and $R_{\text {gal }}=4 \mathrm{kpc}$. For the discussion of the results we define two dimensionless parameters for our systems. $\alpha=R_{\mathrm{pl}} / R_{\mathrm{pl}}^{\mathrm{sc}}=0.02,0.04,0.08,0.12$ 
is the ratio between the scale-length of the single cluster and the scale-length of the super-cluster. $\beta=R_{\text {cut }}^{\text {sc }} / R_{\text {tidal }}=0.2-2.7$ is the ratio between the size and the tidal radius of the super-cluster. Several random realizations of each parameter combination are performed to reduce stochastic errors. The simulations are carried out with the particle mesh code SUPERBOX (Fellhauer et al. 2000) which has the ability to trace many objects with different levels of high resolution subgrids. While the outermost grid covers the whole orbit around the galactic centre with the lowest resolution, the medium sized grids of each star cluster cover the size of the super-cluster and all intermediate interactions between the clusters in medium resolution (10-50 pc), and the innermost high resolution (1 pc) grids cover each single cluster. Both medium and high resolution grids stay focused on the simulated objects and travel with them through the simulation area.

\section{Compact Merger Objects}

A detailed description of the merging time-scales and the efficiency of the merger process will appear in Fellhauer et al. (2001).

The resulting merger objects are compact, spherical and dense objects. They have half-mass radii from $40-150 \mathrm{pc}$ and sizes of $120-1,000 \mathrm{pc}$. The mass of these objects resembles between 75 and $95 \%$ of the total mass of the initial super-cluster. Central densities are derived by fitting exponential profiles to the inner parts. The range of central densities is $100-1,000 \mathrm{M}_{\odot} / \mathrm{pc}^{3}$ with exponential scale lengths of about $10 \mathrm{pc}$. In the outer parts a steep power law $\left(r^{-3.5}\right)$ fits the density best. The surface-density can be well fit with a de-Vaucouleur profile. The velocity dispersion in the central region is about $30 \mathrm{~km} / \mathrm{s}$ but measured along the line-of-sight through the centre one derives about $20 \mathrm{~km} / \mathrm{s}$. All this data suggests that our merger objects have similar properties to nucleated dwarf galaxies $(\mathrm{dE}, \mathrm{N})$. Because the merger process lasts for about a dozen crossing times of the super-cluster (for small $\alpha$ 's this can sum up to more than $1 \mathrm{Gyr}$ ) the merger object is surrounded by several star clusters which are still in the process of merging or are on near-circular orbits around the super-cluster and slowly decaying through dynamical friction. Therefore, our model can account for high specific cluster frequencies $\left(S_{\mathrm{N}}\right)$, which are found for $\mathrm{dE}, \mathrm{N}$-galaxies.

Furthermore, our simple models do not account for destructive processes like tidal heating on eccentric orbits, dynamical friction in the parent galaxy and decay of orbit, shocks due to disc or bulge passages. All these processes would lead to a mass-loss of the merger object and a decrease in its central density. Translated to observations, our objects would transform into $\mathrm{dE}, \mathrm{noN}$ with time, while the number of remaining clusters is also decreasing. Observations show that $\mathrm{dE}, \mathrm{N}$ have higher $S_{\mathrm{N}}$ than $\mathrm{dE}, \mathrm{noN}$.

Our objects also show extended tidal tails along the whole orbit around the galactic centre. Escaped star clusters are found in these tidal features.

\section{Dissolved Merger Object}

In two test calculations the super-cluster was set up on an eccentric orbit $\left(D_{\text {apo }}=60 \mathrm{kpc}, D_{\text {peri }}=30 \mathrm{kpc}\right)$ so that tidal heating could act on the merger 
objects. The merger objects get dissolved with time (mass-loss $\approx 5 \%$ per perigalacticon passage). Due to a disruptive interaction with a second object the central densities drop down from several hundred to $0.3-0.002 \mathrm{M}_{\odot} / \mathrm{pc}^{3}$, but can still be fitted with an exponential in the innermost part. But in the outer parts the material is not bound to the object anymore. It has a "fluffy" structure extending a few kpc. The surface-density can not be fitted with a de-Vaucouleur profile anymore. The measured central velocity dispersions are in the order of $1-3 \mathrm{~km} / \mathrm{s}$, but measured along the line-of-sight through the centre and the outer unbound parts one derives about $10 \mathrm{~km} / \mathrm{s}$. Compared with observations these objects look like dSphs (c.f. Klessen \& Kroupa 1998). Star clusters remaining around these objects can account for the very high $S_{\mathrm{N}}$ of some dSph-galaxies.

\section{Conclusions}

With our simulations we are able to show, that the star clusters in a super-cluster merge efficiently. Comparing our parameters with observations we predict an exponential decrease with time of the number of clusters if the observed superclusters are bound entities.

The resulting merger object has similar properties to $\mathrm{dE}, \mathrm{N}$-galaxies, and if destructive processes are taken into account the next step in the evolution of our objects would be $\mathrm{dE}, \mathrm{noN}$. This also gives a natural explanation why $\mathrm{dE}, \mathrm{N}$ have higher $S_{\mathrm{N}}$ than $\mathrm{dE}, \mathrm{noN}$.

The final stage of the evolution would be a dSph-galaxy, suggesting that at least some of these objects may not have a predicted high dark matter content.

\section{References}

Fellhauer, M., Kroupa, P., Baumgardt, H., Bien, R., Boily, C.M., Spurzem, R., \& Wassmer, N. 2000, NewA, 5, 305

Fellhauer, M., \& Kroupa P. 2000, ASP Conf. Ser., 211, $241^{1}$

Fellhauer, M., Baumgardt, H., Kroupa, P., \& Spurzem, R. 2001, Cel. Mech. \& Dyn. Astron. in press; astro-ph/0103052

Gallagher, S.C., Hunsberger, S.D., Charlton, J.C., \& Zaritsky, D. 2000, ASP Conf. Ser., 211, $247^{1}$

Grebel, E.K. 2000, ASP Conf. Ser., 211, $262^{1}$

Hunsberger, S.D., Gallagher, S.D., Charlton, J.C., \& Zaritsky, D. 2000, ASP Conf. Ser., 211, $254^{1}$

Klessen, R.S., \& Kroupa, P. 1998, ApJ, 498, 143

Kroupa, P. 1998, MNRAS, 300, 200

Mateo, M. 1998, ARA\&A, 36, 435

Whitmore, B.C., Zhang, Q., Leitherer, C., \& Fall, S.M. 1999, AJ, 118, 1551

Zhang, Q., \& Fall, S.M. 1999, ApJL, 527, 81L

1: ASP Conf. Ser. 211: Proceedings of the workshop 'Massive Stellar Clusters' held in Strasbourg Nov. 1999; eds. A. Lancon, C.M. Boily 